\title{
Creación de opinión y percepción sobre China en redes sociales occidentales
}

\section{Creation of opinion and perception about China in Western social networks}

\author{
Fernández Fernández, M., Fernández-Alameda, C. M. y Tardivo, G. ${ }^{1}$ \\ Recibido: 14-05-2019 - Aceptado: 15-08-2019 \\ DOI: https://doi.org/10.26441/RC18.2-2019-A6
}

\begin{abstract}
RESUMEN: Este trabajo se propone explicar la percepción sobre China -potencia en crecimiento-, en redes sociales occidentales y conocer la creación de opinión. Se analizan las noticias más seguidas y comentarios (en BuzzSumo), acerca del país, en 2017. Mediante análisis de contenido se contabilizan shares y commented, se miden indicadores de percepción positiva y negativa, seleccionan palabras más repetidas (Wordclouds.com) y analizan e interpretan los textos. Con aplicación Gephi, se detectan autoridades, influenciadores y centros de debate y opinión. Se realiza también una encuesta a 374 usuarios. El resultado es que la percepción sobre China en redes sociales occidentales varía en función de los temas y de la distancia. Preocupan el poder económico y militar chinos y su realce estratégico, pero se considera a la población pacífica, colaboradora y trabajadora. Las redes funcionan como un cerebro social, en el que los internautas más activos contribuyen al debate y creación de opinión.
\end{abstract}

Palabras clave: análisis textual, ciberespacio, estereotipos, Oriente, Europa

\begin{abstract}
This paper proposes to explain the perception about China and the opinion making in Western social networks. It explores the most commented news (in BuzzSumo) in 2017. Through quantitative and qualitative analysis content, shares, commented and likes are quantified; positive and negative perception indicators are measured; the most repeated words (Wordclouds.com) are selected, and the texts are analysed and interpreted. The monitoring of network users (graphs theory and Gelphi) shows the relationship among them. The triangulation of methods and sources is completed with a survey. As a result, the perception about China on the social networks varies depending on the subject and on the distance. The users show concern about the economic and military power of China, but they consider the Chinese population as peaceful, collaborative and hard-working. The social networks operate as a real social brain, in which the most active users contribute to the debate and opinion.
\end{abstract}

Keywords: textual analysis, cyberspace, stereotypes, Orient, Europe

\footnotetext{
1 Maximiliano Fernández Fernández es Doctor en Ciencias de la Información por la Universidad Complutense de Madrid, Doctor en Sociología por la Universidad Pontificia de Salamanca y Profesor de Teorías de la Información; la Comunicación y Métodos de investigación en la Facultad de Ciencias de la Comunicación de la Universidad Rey Juan Carlos. maximiliano.fernandez@urjc.es, http://orcid.org/00000002-9039-0115
}

Carlos M. Fernández-Alameda es Doctor en Ciencias de la Información por la Universidad Complutense de Madrid y miembro del Cuerpo de Profesores de Secundaria de la Comunidad de Madrid, especialidad en Lengua y Literatura. cfernandezalameda@educa.madrid.org, http://orcid.org/0000-0002-9833-0731

Giuliano Tardivo es Doctor en Sociología por la Universidad Rey Juan Carlos y Profesor visitante de Estructura Social Contemporánea, Sociología del Consumo, etc. en la Facultad de Ciencias de la Comunicación de la Universidad Rey Juan Carlos. giuliano.tardivo@urjc.es, https://orcid.org/0000-00016341-564X 


\section{Introducción: objetivos, marco y estado de la cuestión}

\subsection{Objetivos}

Se pretende en esta investigación desvelar la percepción existente sobre China en las redes sociales occidentales más usuales por tratarse de una gran potencia, cuyas relaciones con Occidente pueden marcar la economía, la política y la cultura de las próximas décadas. Asimismo, mostrar mecanismos de creación de opinión en Internet, como gran cerebro social de inteligencia conectiva, y perfilar el modelo de estas construcciones, en la concepción de modelos de Max Weber. En tercer lugar, encontrar los elementos que definen la percepción sobre un país y las posibles relaciones de causalidad entre esos elementos y la imagen construida en las redes. Se buscará medir estas magnitudes intangibles a través de variables observables.

Siguiendo la recomendación de Wright Mills en La imaginación sociológica (1959), se busca responder a cuestiones del tipo ¿qué se dice en las redes sociales occidentales acerca de China?, ¿qué opinan sobre China 374 usuarios de redes entrevistados?, ¿cómo se crea opinión mediante inteligencia conectiva en Internet (¿qué redes, quiénes escriben, de qué manera se crea opinión, qué intereses pueden subyacer, qué tópicos persisten, se hacen análisis serios y documentados...?), ¿qué lugar ocupa China en el ciberespacio?, ¿qué noticias positivas se publican y qué noticias negativas?, ¿con qué expresiones o términos, favorables o desfavorables, se alude y cuáles predominan?...

A partir de los avances en el estudio de redes sociales durante los últimos años y en la construcción de la imagen sobre China en Occidente cabe señalar una serie de paradigmas y corrientes principales, a las que se vincularán algunas de las hipótesis de esta investigación para contribuir a verificarlas o rectificarlas, mientras que otras son resultado de experiencias, intuiciones y conjeturas propias.

\subsection{Marco teórico}

Existe una larga tradición sociológica y comunicológica que acepta la existencia de una conciencia colectiva (Durkheim, 1893), alma colectiva (Le Bon, 1895), mente colectiva y sistema de relaciones entre las mentes individuales (McDougall, 1912), una noosfera (Vernadski, 1926), memoria colectiva (Halbwachs, 1939), espacio virtual de la psiquis (noogénesis) o esfera del entendimiento, del Nous (Theilhard de Chardin, 1950), etc., que contrasta con autores que priorizan la intencionalidad subjetiva, como Max Weber (1905) o Sigmund Freud (1921), defensor del inconsciente individual.

Aparte de esta tradición interdisciplinar y focalizando más en los medios de comunicación, entre los que hoy cuentan de forma muy activa las redes sociales, Elihu Katz y Paul Lazarsfeld (1955) observaron ya que los medios influyen en la opinión pública a través de grupos o líderes de opinión, entre los que ahora deben incluirse de forma destacada los llamados influencers o influenciadores. Asimismo, Edwin Hutchins, en la Universidad de California, defendía a finales de los 80 que el conocimiento se comprendía y organizaba mejor cuando se analizaba como un fenómeno distribuido, como una comunidad de inteligencia extendida (Timoteo Álvarez, 2013, p.16). De alguna forma podemos equiparar este conocimiento colectivo al que se produce en las redes sociales (M. Fernández, 2008), en una conexión de neuronas, incluidas las neuronas espejo, localizadas en el cerebro humano en 1996 a través de la Resonancia Magnética Funcional (RMF). No es sólo una metáfora, se trata de compartir y extender ideas en las redes sociales, incluso sentimientos. Como explican Ángel Rubio y Pablo Sapag (2013, p. 96), las áreas del cerebro humano que contienen dichas neuronas también comunican 
con el sistema límbico "facilitando así la conexión con los sentimientos de otra persona a través del reflejo de dichos sentimientos".

Es más, Timoteo Álvarez (2013, pp. 14-15) sostuvo que existe un mundo real que puede verse desde la perspectiva de la teoría de las redes, ideas o "memes", en terminología de Dawkins, que saltan de cerebro en cerebro a través de un proceso como el de las neuronas espejo. Se produce, como decía Richard Dawkins (1976), un efecto imitación, a través de la implantación de palabras, frases hechas, melodías, refranes, imágenes, "memes" ... "Esta hipotética consciencia social -añade Timoteo- se manifiesta en forma de "inteligencia conectiva"”.

En los últimos años, Derrick de Kerckhove (2010) formulaba su inteligencia en conexión, considerando la inteligencia conectiva o "a escamas" como una fase superior a la de inteligencia colectiva, múltiple o distribuida. Rodríguez, Baños y Arroyo (2011, p. 4) subrayan que "personas, organizaciones y gobiernos en todo el mundo están incluyendo sus comunicaciones a través de estas plataformas web" y tienen ya nichos influyentes en las redes sociales de mayor alcance, como Facebook, Twitter y YouTube.

Las redes sociales, expresión introducida por los antropólogos británicos Alfred Radcliffe-Brown y John Barnes, han sido definidas como "una estructura social formada por personas o entidades conectadas y unidas entre sí por algún tipo de relación o interés común", tal y como figura en el Estudio Anual de Redes Sociales 2018, de IAB Spain. También se ha destacado que las comunidades on line son fuente de discursos diversos que expresan tendencias dominantes en la sociedad (Armony y Velásquez, 2015, p. 131) y que permiten hablar de una ciudadanía digital, en la que los millennials son actores principales que demandan mayor implicación incluso desde instancias educativas (García Galera y Fernández Muñoz, 2016).

De esta forma, en las redes sociales se crea opinión y se comparten ideas, sentimientos, emociones, afectos... y también estereotipos, recelos, desconfianza hacia otras culturas... un mundo de percepciones. Lo señalaban asimismo, entre otros autores, M. Fernández (coord.) en Comunicación en la sociedad red: la construcción mediática de la realidad (2008) y en "La diversidad y la discriminación en encuadres de El País, El Mundo y Abc" (2013: 91-106), así como Javier Serrano-Puche (2016, p. 19) en la revista Comunicar: "La red no sólo despierta emociones en sus usuarios y sirve de cauce para la expresión de los afectos, sino que también influye en el modo en que dicho afecto se modula y despliega, así como en la configuración de la identidad de la persona".

Como escriben asimismo Ariel Armony y Nicolás Velásquez (2015, p. 130), a propósito de las comunidades virtuales latinoamericanas, "los canales de los medios sociales pueden abrir una ventana a una multiplicidad de puntos de vista expresados por individuos. Twitter, Facebook y muchos otros medios nos permiten estudiar las expresiones espontáneas de opinión compartidas públicamente por los individuos".

La cuestión que se dilucida aquí también y que comprobaremos en nuestro estudio de redes es si estos canales propiciados por las TIC funcionan en una horizontalidad democrática, como se ha venido sosteniendo desde finales del siglo XX (Norris, 1999; Negroponte, 1995; Rheinghold, 1993...) o, como ha puesto de manifiesto Torres Nabel (2015), no se produce tal horizontalidad y existen una serie de factores (contexto, organización, estructura de las redes) y actores (centrales, secundarios y meros puentes) con diferente influencia, que actúan como "programadores" o como "enlaces" con capacidad respectivamente para construir redes o para conectarlas. 
En la misma línea y para el caso concreto de la televisión, se posicionan autores como Golan (2010), quien subraya los aspectos geográficos, culturales y jerárquicos; Martínez Lirola $(2014,2016)$, que destaca la influencia de los medios en la construcción de la imagen de los otros cuando no existe un contacto cercano, o como RodríguezWangüemert, Rodríguez-Breijo y Pestano-Rodríguez, quienes han constatado la valoración mayoritariamente negativa en informativos de televisión acerca de China (2019: 134), o la problematización de su imagen por un recurso excesivo a la violencia (2017: 983).

Las redes sociales, al ser más democráticas y abiertas, diversifican mucho más las miradas de los otros y sobre los otros y contribuyen más al conocimiento de la alteridad.

\subsection{Contexto: Las redes sociales en la actualidad y la percepción sobre China}

En este marco, han proliferado en los últimos años, aparte de los trabajos mencionados anteriormente, estudios como el que analiza la influencia del tipo de cobertura que los medios ofrecen sobre China en la toma de decisiones de funcionarios de la Unión Europea (Li Zhang, 2010); estudios como el de Axel Bruns, Tim Highfield y Jean Burgess sobre el papel de Twitter en la llamada "primavera árabe" en 2011 a través de hashtags, o el dirigido por Jesús Timoteo (2013) sobre neurocomunicación y gestión de la comunicación social basada en neurociencias. Se ha analizado el "pánico integracional" en la Unión Europea (Cross y Ma, 2015) y la comunicación de los jóvenes en el mundo digital (García Galera y Fernández Muñoz, 2016). Y se ha analizado la influencia negativa en la construcción de imagen sobre China de encuadres televisivos sesgados por Rodríguez-Wangüemert, Rodríguez-Breijo y Pestano Rodríguez (2017 y 2019).

En la actualidad alrededor de 4.000 millones de personas en todo el mundo (53\%) tienen acceso a Internet. Facebook, presente en todos los países, superaba en 2018 los 2.170 millones de usuarios; Youtube, los 1.500; Whatsapp, como red de referencia para las relaciones sociales, cuenta con unos 1.300 millones de usuarios, al igual que Messenger, según el informe Digital, Social \& Mobile in 2018. Para conocer las posibilidades de creación de opinión, es importante el dato revelado por el estudio, según el cual un $85 \%$ declara que sigue a influencers a través de redes sociales, alcanzando el $71 \%$ el porcentaje de los que sólo leen y 29 los que leen y comparten. En estos casos, las redes más utilizadas son Facebook (63\%), Twitter (34\%) y YouTube $(20 \%)^{2}$.

En China, si bien existe una variedad de "weibos" como Tencent Weibo o Sohu Weibo, según DMR, actualmente las más populares son Qzone, con 568 millones de usuarios activos, y Sina Weibo, lanzado en agosto del 2009 con características similares a las de Twitter y de Facebook y que cuenta con 376 millones de usuarios activos.

Joshua Cooper Ramo (2007) muestra que las visiones de China en el mundo están polarizadas: entre quienes la ven como un país que ama la paz, que coopera en la esfera internacional pero que es un actor autónomo, y la visión de China como un país militarizado, autoritario y con una fuerza que obstruye las decisiones internacionales.

Los resultados de un estudio realizado por investigadores de la Universidad de Harvard sobre redes sociales chinas muestran que el Gobierno, además, "bombardea las redes sociales con 488 millones de comentarios cada año"3.

\footnotetext{
${ }^{2}$ http://www.iabspain.net.

${ }^{3} \mathrm{http}: / /$ tecnologia.elpais.com/tecnologia/2016/05/24.
} 
La percepción occidental sobre China se habría deteriorado desde 2010 como consecuencia del "dramatic economic ascension of China" o "China's economic ascension", según datos del Transatlantic Trends Survey de 2010, realizado por la German Marshall Fund.

En 2014, el Pew Research Center de Washington publicó una encuesta a 48.643 personas en 44 países, que resultó favorable a China, con un $49 \%$ de respuestas favorables frente a un $32 \%$ de respuestas contrarias. La opinión europea y estadounidense sobre China era ligeramente desfavorable, aunque con excepciones, como Reino Unido y Grecia. Se equilibran las cifras a medida que se produce un acercamiento geográfico con resultados positivos en Ukrania, Rusia, Túnez, Palestina... y negativos en Israel, Egipto, Jordania, Turquía... Mejora mucho en el entorno (Pakistán, Bangladesh, Malasia, Tailandia, Indonesia, Corea del Sur), pero se invierte en Filipinas, India, Vietnam, Japón... En América Latina predomina la opinión favorable, según Pew Research Center ("Ratings for China Generally Favorable. Views of China", Spring 2014 Global Attitudes survey. $Q 15 b)^{4}$. El informe Transatlantic Trends confirma esta tendencia. El $80 \%$ de los españoles consideraba "no deseable" un liderazgo mundial de China. E1 55\% tenían una opinión contraria mientras que sólo el 35\% expresaba ideas pro China. En cambio, en un estudio publicado por Arroyo-Almaraz y Van-Wyck en la Revista Latina de Comunicación (2011, p. 490) se asegura que los españoles opinan que "la población asiática, mayoritariamente procedente de China, es la más dinámica y emprendedora de todas las poblaciones inmigrantes" en el país.

Según Soo Yeon, Sophie Meunier y Zsolt Nyiri (2016), en Europa existe una correlación entre la percepción negativa sobre Estados Unidos y sobre China. El enfoque de su trabajo, diferente al presentado aquí, ya que se centra en el estudio de esa potencial relación de interdependencia entre China y Estados Unidos sobre la opinión pública europea, nos mueve a contrastar en las redes sociales algunas de las afirmaciones que extraen de su estudio, como la relativa al temor y rechazo que los europeos tendrían hacia el poder económico de Pekín.

En un estudio similar al nuestro, en el ámbito latinoamericano, publicado por Ariel C. Armony y Nicolás Velázquez, en la revista Nueva Sociedad en octubre de 2015, se asegura que "la acelerada expansión de la presencia china en América Latina ha forzado a las dos partes a hacerse una rápida idea del «otro»".

Aunque encuestas de opinión pública indican que América Latina percibe favorablemente el auge de la República Popular China, "este encuentro ha generado una reconstitución de las identidades, lo que deriva en imaginarios basados tanto en experiencias previas como en prejuicios". Armony y Velásquez, para conocer la construcción de estas percepciones, analizaron comentarios de usuarios de Facebook, suscitados por noticias publicadas entre enero de 2013 y septiembre de 2014 en ocho diarios líderes en Argentina, Chile, Colombia, México y Perú con el objetivo de entender los conceptos que estructuran las visiones negativas sobre China en América Latina y reconstruir sus discursos.

\subsection{Hipótesis}

Según los citados estudios previos y datos aportados, que no recapitulamos para no repetir, o, dicho de otra forma, a partir de los paradigmas o corrientes recogidas en este marco teórico y de este contexto, intentamos contrastar en primer lugar si efectivamente se crea opinión en redes sociales y participan influenciadores o internautas que generan opinión y debate. En consecuencia, formulamos como hipótesis, de tipo deductivo y

\footnotetext{
${ }^{4}$ http://www.pewglobal.org/2014/07/14/.
} 
convalidables, las siguientes: 1.: "En las redes sociales confluyen agentes institucionales e influenciadores libres, que crean opinión y generan debate y usuarios independientes y ocasionales, que no llegan a implicarse en las redes". La segunda hipótesis (H.2.) pretende contrastar el uso que se hace de las principales redes: "Facebook es la red preferida entre las occidentales y la que suma mayor número de engagements en cuestiones de interés político internacional con bastante distancia sobre las demás". La siguiente (H.3.) plantea la veracidad e interés de lo publicado en redes sociales occidentales: "Los contenidos más seguidos en redes sociales occidentales son interesantes y generan debate de ideas". La cuarta, en la línea de los estudios de Armony y Velasquez sobre percepciones acerca de China en América Latina y de la encuesta del Pew Research Center de Washington a 48.643 personas en 44 países, apunta a la ambivalencia de la influencia en Internet, que puede ser tanto positiva, contribuyendo al mejor conocimiento y comprensión entre países, como negativa, aumentando artificialmente la alarma por cuestiones no tan preocupantes. Dicho de otra forma (H.4.): "Los medios y agentes que operan en Internet ejercen influencias ambivalentes, contribuyendo tanto al mejor conocimiento $y$ entendimiento entre países (influencias integradoras) como a generar desinformación, desconfianzas y recelos mediante comentarios alarmistas, uso de estereotipos, etc. (influencias desintegradoras)". Finalmente, la quinta hipótesis alude a la incidencia de los temas y de la cercanía/lejanía geográfica en la percepción, en la línea de lo indicado por Golan (2010), quien subraya los aspectos geográficos, culturales y jerárquicos, y por Rodríguez-Wangüemert, Rodríguez-Breijo y Pestano-Rodríguez (2019: 134), quienes destacan la incidencia de los medios en la construcción de imágenes acerca de los otros. La quinta hipótesis, por lo tanto, apunta a que (H.5.): "La percepción sobre China en las redes sociales occidentales varía en función de los temas planteados, siendo más positiva acerca de la laboriosidad y capacidad emprendedora de la población y más negativa cuando se valoran los efectos de su competencia industrial o de su poder económico y militar. Y varía asimismo en función de la proximidad".

\section{Metodología: unidad de análisis, diseño, métodos y técnicas}

\subsection{Unidad de análisis, triangulación metodológica y técnicas}

La unidad de análisis está constituida por las diez noticias más seguidas sobre China en redes sociales occidentales (Facebook, Linkedin, Twitter, Pinterest, Google+) durante los meses de septiembre, octubre y noviembre de 2016, en selección de Buzzsumo, cerrada a fecha de 23-11-2016, con sus correspondientes respuestas y comments más significativos; en total 401 comentarios de la más diversa procedencia (Estados Unidos, Europa, Filipinas, India, Pakistán...), de los que nos interesan los que aluden directamente al gigante asiático.

Se analizan estas noticias y comentarios mediante triangulación de métodos y fuentes, usando técnicas cuantitativas y cualitativas: cómputo de shares y engagements en las principales redes, desde Buzzsumo; localización y significación de palabras más repetidas a través de Wordclouds, y de palabras clave; análisis e interpretación de los textos valorando percepciones sobre China positivas o negativas; seguimiento de creación de opinión en redes y elaboración de un grafo social, siguiendo la teoría de grafos, rama de las matemáticas discretas, utilizando la herramienta Gephi, que representa las relaciones entre usuarios. 


\subsection{Aplicación de la teoría de los grafos}

La teoría de grafos, que se aplicará al conocimiento de los internautas más influyentes, se está extendiendo en el campo de las Humanidades Digitales para estudiar las relaciones entre personajes en una obra literaria, dramática o cinematográfica. Su origen se remonta a la resolución del problema de los siete puentes de Köninberg (actual Kaliningrado) que propuso Leonard Euler (1741). Tal y como explica Gerard Alexanderson (2006), el científico suizo planteó un dilema muy popular entre los habitantes: ¿Es posible atravesar la ciudad sin pasar dos veces por el mismo puente? utilizando lo que Leibniz llamó "geometría de posición". Para Euler, fue la disciplina que mejor podía solucionar la cuestión ya que "no implicaba distancias, ni cálculos con las mismas". De hecho, lo importante es la relación entre nodos y aristas. Es decir, entre las zonas (nodos), o islas de la ciudad que separaba el río y los puentes (aristas). Aunque la conclusión de Euler fue que no había forma de resolver el problema a no ser que cada nodo tuviera un número par de aristas, dio origen a todo un campo de investigación.

Este tipo de logaritmos ha resultado ser muy útil para la creación de redes sociales digitales como Facebook, Twitter, Youtube, o ResearchGate. La evolución de la analítica de redes sociales nos ha llevado a poder representar de forma gráfica las relaciones de amistad en el mundo virtual. Pero podríamos aplicarlo también a nuestras relaciones en la vida real. La famosa red de contactos profesionales Linkedin utiliza la hipótesis de los seis grados de separación desarrollada por Duncan Watts (2004). Según su modelo, cualquier persona está conectada con el resto del planeta a través de una red de conocidos que no alberga más de cinco intermediarios. Es decir, sólo seis enlaces serían suficientes para acceder a una enorme cantidad de contactos. Este crecimiento exponencial se vería multiplicado aún más por la disponibilidad del usuario actual de tecnología muy avanzada. Como herramienta informática ha sido elaborada por un equipo internacional de matemáticos y ha sido presentada y descrita por Mathieu Bastian, Sebastien Heymann y Mathieu Jacomy (2009).

\subsection{Técnicas para la comprobación de hipótesis y análisis del mensaje}

En consecuencia, para comprobar las cinco hipótesis planteadas se recurre a cuantificar y seguir las conexiones entre los usuarios más activos e influenciadores, elaborando un grafo social, con la herramienta Gephi para su mejor visualización (H.1.); a cuantificar shares y engagements (H.2) de cada una de las redes más utilizadas en las diez noticias más compartidas; a analizar los contenidos de cada una de las noticias y comentarios para valorar el interés de lo publicado (H.3.); a detectar si contribuyen a un mejor conocimiento y entendimiento entre países o a crear alarmismo y divulgar estereotipos (H.4.), y a realizar estudios cuantitativos y cualitativos de contenidos para sopesar las percepciones positivas y negativas sobre China que genera cada una de las noticias y las cargas semánticas que conllevan, utilizando en su medición una escala de Likert. Para el análisis de los términos más repetidos, aparte del enfoque contextual, se recurre a la nube de palabras. Y, además, se señalan términos y expresiones clave (KWICV o Key Word in Context), en la idea de que resulta más objetivo e ilustrativo estudiarlas en su contexto, siguiendo las técnicas utilizadas por Duojiezhaxi (2016: 351), quien propone analizarlas junto con las más repetidas y las llamadas nexos o sin significado.

Recurrimos también a categorías e indicadores para un análisis comprensivo e interpretativo de los mensajes, como los de carácter integrador/desintegrador de los comentarios en redes, buena/mala calidad de los productos, colaboración/dominación, etc. Son categorías e indicadores similares a los utilizados en otras investigaciones, como 
las de Valkenburg, Semetko y De Vreese (1999), quienes emplean variables de responsabilidad, interés humano, conflicto, moralidad y consecuencia económicas para definir encuadres informativos. Carmen Rodríguez-Wangüemert, Vanessa RodríguezBreijo y José Manuel Pestano Rodríguez (2017) utilizan categorías como cooperación, confrontación, amenaza, potencia y oportunidad en sus estudios de encuadres sobre China en 2017 y los de "capacidad, potencial y desarrollo", "penumbra, sombra y oscuridad", "entorno peligroso" y "organización exótica", en 2019. En otros casos, se crea un índice de imagen transmitida por los deportistas analizando atributos positivos y negativos, contenidos subyacentes, imágenes del jugador, etc. (Fernández-Alameda, 2011) o se cuantifica, compara y analiza contenidos con referencias explícitas a integración, diversidad, desigualdad y homofobia (M. Fernández, 2013).

\subsection{La aportación de la encuesta}

Finalmente, el aporte de la encuesta permite contrastar la comprobación de las hipótesis, en concreto, la idea que tienen los propios internautas sobre su función y la de otros participantes en la red (H.1.), sus preferencias por unas u otras redes (H.2.), su confianza e interés por los temas abordados (H.3.), la propia valoración positiva o negativa de su influencia en redes (H.4.) y la opinión que expresan sobre China y su población en función de los temas planteados (H.5.).

Se realiza la encuesta en inglés y español a usuarios de redes sociales elegidos por muestreo intencional y utilizando la herramienta de Google Forms (enlaces http://bit.ly/Form-China-Spain, en español, y http://bit.ly/Survey-China-Spain, en inglés), para conocer sus opiniones y actitudes sobre redes sociales y sobre China. El cuestionario fue respondido, hasta el 13 de mayo de 2019, por 377 usuarios (362 en español y 15 en la versión inglesa), de ellos, 142 hombres (38\%) y $230(61,5 \%)$ mujeres; la mayoría $(67,8 \%)$ menores de 25 años, seguidos de los comprendidos entre 25 y 50 $(17,7 \%)$ y de los mayores de $50(14,5 \%)$. El mayor número de respondentes tiene estudios universitarios $(60,2 \%)$ o de Bachillerato $(22,6 \%)$, mientras que un $14,5 \%$ ha alcanzado el postgrado. Respecto a la situación laboral, el $32,2 \%$ son empleados y autónomos $(5,9)$, mientras que el 41,6 estarían desempleados, jubilados o estudiando ${ }^{5}$.

La mayoría de los participantes en la encuesta (no los que intervienen en redes, que proceden de todo el mundo), son españoles, aunque también hay personas que contestan desde México, California, Irlanda, República Dominicana, Colombia, Boston... ${ }^{6}$

El orden de las preguntas del cuestionario ha sido el siguiente: 1. Datos demográficos del encuestado (iniciales o seudónimo, identidad en la red y lugar de residencia, sexo, edad, nivel de estudios, situación laboral. 2. Frecuencia en el uso de redes sociales. 3. Redes más utilizadas (hasta un máximo de tres). 4. Influencia recibida de otros usuarios. 5. Forma de participación e influencia propia ( $\mathrm{n}^{\mathrm{o}}$ de seguidores). 6. Tipo de influencia propia (integradora/desintegradora). 7. Aceptación de la influencia de las redes. 8. Opinión sobre China (emprendedores, pacíficos, potencia económica y militar y calidad de sus productos). 9. Independencia de las redes occidentales. 10. Independencia de las redes

\footnotetext{
5 (https://docs.google.com/forms/d/1AMZ-qQZUHljBVMMY4FUaxUOYS7PyIsxcln1phLgguw4/edit\#responses).

${ }^{6}$ (https://docs.google.com/forms/d/1 AMZ-qQZUHljBVMMY4FUaxUOYS7PyIsxcln1phLgguw4/edit\#responses y https://docs.google.com/forms/d/1_dymbZZ9bQPfgkD1ZVJrd9DGOs-65aRBSbtcm1QHzjE/edit\#responses
} 
chinas. 11. Interés y veracidad de lo publicado en redes occidentales. 12. Interés y veracidad de lo publicado en redes chinas. 13. Otras observaciones y comentarios.

\section{Análisis y resultados}

\subsection{Análisis de las noticias y comentarios}

La preliminar investigación exploratoria de la unidad de análisis, constituida por las diez noticias más seguidas en redes sociales occidentales, presenta los siguientes resultados:

La primera noticia, de Daily Koss (16-9-2016), "Busted! The new Trump hotel in D.C. is filled top-to-bottom with goods made in China", la más seguida, con 270.900 compartidos o participaciones (shares o engagements) y 312 comentarios (commented) es una crítica muy dura al entonces candidato a la presidencia y actual inquilino de la Casa Blanca, Donald Trump, por tener productos chinos y, en menor medida, de otros países, en sus hoteles. Se le censura - la mayor parte de los internautas son americanos- por comprar productos "made in China" y "hacer grande a China" (parafraseando su eslogan de campaña) en lugar de a Estados Unidos. La gran mayoría de las expresiones clave, tanto en la noticia como en los comentarios de los internautas, referidas al país asiático ("hecho en China" o "salarios bajos como en China", "productos de China", "trabajos en China"), están cargadas de connotaciones negativas. En la escala de -2 (muy negativa) a +2 (muy positiva), la calificamos como bastante negativa (-1), ya que el fuerte de las críticas recae en Trump.

La segunda noticia, de Local Press (8-10-2016), "Bihar court becames $1^{\text {st }}$ in India to ban sale of 'Made in China' products", con 204.100 engagements y shares y 17 commented, alude a que la corte de Bihar, en la India, es la primera en prohibir productos del gigante, después de declararlo enemigo por apoyar a Pakistán en su conflicto fronterizo. Los internautas, en su mayoría de la India, apoyan la decisión ("All importers and exporters should follow this and should stop all trade business with China"), critican la calidad de los productos chinos ("their products are truly sleazy") y animan a no comprarlos ("every individual person should reject Chinese goods automatically and importers will stop importing goods from China however cheap it may be") para acabar con la hegemonía comercial del país ("the first step to stop Chinese commercial hegemony"). Debido al enfrentamiento fronterizo, aluden a China como enemigo ("China enemy" y "our enemies"). La percepción en este caso es muy negativa (-2).

También el tercer contenido, de Newsweek (3-10-2016), "How Donald Trump ditched U.S. steal workers in favor of China", con 169.900 participaciones y 8 comentarios, carga directamente contra Trump e indirectamente contra Beijing por la intención del ahora mandatario americano de prescindir de trabajadores del acero estadounidenses en favor del competidor, país, según Newsweek, "cuyas prácticas han ayudado a diezmar la una vez poderosa industria central de Estados Unidos". El periódico acusa a Trump de dañar la industria propia y beneficiar a la competencia ("they are providing financial benefits to an array of Chinese companies and even the government") y a sus bancos, que son brazos del partido ("arms of the state, tightly controlled by the Chinese Communist Party and provide financing to companies that are competitors to American manufacturers in other industries"). Los internautas, contribuyendo a la creación de opinión, refuerzan esas críticas sobre la "inundación" de acero chino ("China has been flooding the world with cheap steel for years"). La percepción de los usuarios, mayoritariamente de origen americano, es muy negativa (-2), porque cargan duramente contra los productos y manufacturación del gigante asiático. 
La cuarta noticia, de Mens XP (31-10-2016), sobre una chica que obtiene 20 iphone7 de sus novios y los vende en una web para comprar una casa ("Girl gets 20 iPhones from 20 boyfriends, sells them off to buy a house in China"), recibe 108.200 participaciones y 14 comentarios. Al tratarse de un asunto de menor calado, los comentarios se centran en si lo que obtiene le puede servir acaso para el primer pago de la casa. No aparecen, en consecuencia, valores significativos que puedan incidir en una percepción positiva o negativa, cómo mucho "It's China, everything is posible", por lo que la situamos en el centro de la escala de Likert (neutro o indiferente).

De forma similar, aunque por razones diferentes, valoramos la quinta noticia, publicada por The New York Times (9-10-2016), "An open letter to the woman who told my family to go back to China", que tiene 107.100 compartidos y 8 comentarios. La carta, que firma Michael Luo, "asio-americano", está dirigida a la señora que gritó a un grupo de asiáticos en Manhatan: "Go back China [..] go back to your fucking country", lo que califica como insultos racistas. Menciona que otras muchas personas contaron experiencias similares, lo que reafirman algunos internautas: "I've been shouted this many times, even in supposedly 'post-racial' Hawai", añadiendo que no se han sentido bienvenidos ("even in this 'multicultural' NYC-I've felt less and less welcome"), que son extranjeros ("we are foreign. We are not American") y que algunos son orientales, pero no de China ("We're not from China"). Se acusa incluso de racismo al corresponsal de Fox "for China towns interviews"). En contrapartida, el propio alcalde de Nueva Yok, Bill de Blasio, asegura que los que no pertenecen a la ciudad son quienes hacen estos comentarios ("What doesn't belong here are comments like you heard today"). En la valoración se equilibran los comentarios racistas o xenófobos, vertidos en expresiones clave, a los que se alude con la condena de los mismos.

En la sexta información, "Duterte kinasuhan si Trillanes ng treason for contacting China and giving them power over Scarborough Shoals", publicada por Breaking News (19-112016) y que cuenta con 86.500 shares, no aparece comentarios. El autor, Edwin Tulfo, periodista filipino, se hace eco de que el alcalde de Davao y presidente de Filipinas, Rodrigo Duterte, acusa de traición al senador Antonio Trillanes por contactar con Beijing, visitar el país 16 veces en 2012 y darle poder sobre los Scarborough Shoals (Bancos de Arena) filipinos en el mar del Sur de China. Se acusa al gigante asiático de rellenar y de construir estructuras ("to fill up the waters and built structures") en los bancos de arena del Mar del Sur. Se alimenta la imagen sobre China como potencia expansiva que se aprovecha de una "traición" y ocupa espacios que en principio no le pertenecen, por lo tanto, bastante mala (-1).

De carácter opuesto es la noticia 7, correspondiente al medio pakistaní Voice of Pakistan (22-9-2016), titulada "If China and Pakistan Army Attack On India, What will happened with Hindu's Indian Media Reporting" y que cuenta con 86.400 participaciones y 37 comentarios. La noticia se presenta en un vídeo en hindi. Se plantea un posible ataque a la India de las armadas china y pakistaní en el territorio de Cachemira. Los internautas se alinean también en el sentido de la información reafirmando las condenas a la India y la amistad con China ("China dosti", "Pakistan China dosti zinda abad", Pak-Chaina dosti zindabad). Pero también se deslizan algunas críticas al hecho de que China no permita rezar abiertamente a los musulmanes ("China does allow muslims to pray openly") y a que suprima derechos ("China suppresses the rights of muslims and does not even allow to offer prayers in open"), por lo que la noticia se valora de forma neutra.

El contenido 8, "Ched: Mandarin to be part of elementary, high school and college currículum as preparation for Ph-China partenership", publicada por Newsph, el 20-102016, con 80.300 shares y 13 comentarios, es uno de los pocos casos en que los 
internaturas no siguen la línea de creación de opinión del medio oficial y muestran escepticismo. Según Newsph, en clara línea oficialista, Filipinas se propone añadir el mandarín en la formación de profesionales que colaboren en el futuro con el país vecino, considerándolo conveniente para la mayoría de la población por el aumento de las inversiones y los negocios con China, de donde proceden los futuros jefes. Los internautas rechazan ese optimismo, consideran que la información es falsa, niegan que Filipinas sea una colonia de China ("we are not a fracking chinese colony ffs") y aducen que el idioma es difícil ("which is one of the most difficult language") y que en los países vecinos en los que se ha implementado, el resultado ha sido devastador: "there are countries, like Malaysia, Indonesia, Thailand... they have already implemented it in their school curriculum for several years, but the result is devastating". Y que es mejor producir calidad que cantidad ("we can produce good quality not the worse quantity"). En definitiva, el estudio de las expresiones clave permite detectar que existe un contraste entre el entusiasmo del medio, muy oficialista, y el escepticismo de los internautas, por lo que el resultado es equilibrado (0).

El noveno contenido, "Duterte to bring home \$24 billins from China", divulgado por GMA News Online (21-10-2016), que logra 77.200 shares y 4 comentarios, dos de ellos en tagalo, se hace eco de la visita del presidente filipino, Rodrigo Duterte, a China y de la captación de 24 billones de dólares para inversiones y créditos, lo que puede generar 2 millones de trabajos. Los cuatro comentarios de los internautas no aluden a la imagen de China, aunque alguno se pregunta si son solo inversiones ("Promise of investments pa lang po" La percepción es positiva (+1) porque el Estado aparece como una gran potencia económica capaz de realizar grandes inversiones en países vecinos como Filipinas.

Finalmente, la décima noticia, publicada por Frontera, 16-10-2016, titulada "Why Duterte will begin buying Weapons from China and Russia in Non-US", con 76.800 compartidos y sin comentarios, alude a la posible decisión del presidente filipino, Rodrigo Duterte, de comprar armas chinas y rusas en lugar de americanas, para hacer frente a los ataques terroristas. La noticia contribuye a una percepción negativa de China, porque, aunque el presidente filipino parece inclinarse por sus armas, también se asegura que Pekín y Moscú se están moviendo "agresivamente" en el mercado mundial de armas ("Russia and China are agressively moving to capture larger shares of the region's spending"), generando tensas relaciones ("strained relations"), y criticando la situación del Mar del Sur, conocido como "The second Persian Gulf". Además, preocupa a Filipinas que su vecino esté construyendo islas en la zona ("the Philippines are most concerned with possibility that China will start building islands just 220 miles northwest of Manila on the Scarborough Shoal"). Se recuerda que China y Rusia tienen una larga historia de conflictos fronterizos ("China and Russia have a long history of conflict and suspicion over adjoining territory"). La fotografía, del presidente filipino observando armas, en medio de un grupo grande de militares, resulta igualmente preocupante (-1).

El aporte de las imágenes, en consecuencia, resulta también contrario al país: las noticias 1 y 2 presentan productos en los que se lee "made in China", como motivo de las críticas; la $3^{\mathrm{a}}$ a un Donald Trump acusado por la prensa de beneficiar a China en perjuicio de la industria americana y la $10^{\mathrm{a}}$ al presidente filipino, entre armas y militares, decidido a comprar más armas a China, en medio de conflictos. Tienen una significación más neutra las imágenes de la noticia 4 (iphone7) y 7 (presentadora de informativos). Por lo demás, la inexistencia de otras imágenes (informaciones 5, 6, 8 y 9) denota escasa atención en la red al elemento informativo y opinativo de carácter gráfico. 


\subsection{Verificación de hipótesis}

\subsubsection{Creación de opinión: de los medios a los influenciadores}

Para corroborar la primera hipótesis sobre los agentes que intervienen en las redes sociales, cabe observar en primer lugar que las diez noticias más compartidas en redes sociales occidentales en septiembre, octubre y noviembre de 2016 son emitidas por medios de comunicación. En este caso los más seguidos fueron Daily Koss (270.900 shares), Local Press Co (204.100), Newsweek (169.900), Mens XP (108.200), The New York Times (107.100), Breaking News (86.500), Voice of Pakistan (86.400), Newsph (80.300), GMA News Online (77.200) y Frontera (76.800). Son medios en algunos casos independientes y críticos y en otros defensores de posturas gubernamentales (no comprar productos chinos, apoyo chino-pakistaní frente a la India, defensa del aprendizaje del mandarín...). En consecuencia, cabe concluir que los primeros influenciadores, más que los gobiernos e instituciones y también más que los internautas independientes, siguen siendo los medios de comunicación, que informan y comentan los temas e inician la creación de opinión, ya que la mayoría -aunque no todos- de los comentarios posteriores abundan en las mismas direcciones. La oposición a esos comentarios es mayor cuanto más se aproximan al enfoque institucional o más se convierten en portavoces de los gobiernos. A partir de aquí, tiene considerable peso también la incidencia de agentes individuales muy activos que se convierten en verdaderos núcleos o centros desde los que se irradia opinión y se genera debate (authorities). Esto permite actualizar la teoría de los dos pasos de Paul Lazarsfeld y Elihu Katz, quienes destacan la influencia de los grupos intermedios y de los líderes de opinión, en el sentido de que actualmente los líderes y grupos intermedios son los influenciadores de las redes sociales. Dicho de otra forma, los internautas inciden muy mayoritariamente en las opiniones que expresan los medios en sus informaciones, por lo que, auque a veces se genera debate, puede afirmarse que casi siempre crean opinión en su línea informativa y editorial. Cabría pensar que los medios puedan ocultar comentarios contrarios a su línea, pero resultaría extremadamente complicado de demostrar y aparecerían esos textos adversos en los competidores. Sin embargo, a tenor de los resultados electorales, hay que concluir que ni medios ni internautas consiguieron que Trump perdiera las elecciones.

Para representar la red de comentarios de los internautas a la noticia más seguida y comentada en los meses de septiembre a noviembre de 2016, según la red Buzzsumo, "Busted! The new Trump hotel in D.C. is filled top-to-bottom with goods made in China", que es la que más creación o debate genera, hemos utilizado la teoría de grafos, rama de las matemáticas discretas. En el caso que nos ocupa, se trata de representar las relaciones entre los comentarios a la noticia. Es decir, se pueden dar varios casos: a) usuario que escribe espontáneamente sin hacer referencia a ningún comentario, b) usuario que responde o añade información a otros, y c) usuario que responde a un mensaje anterior, que a su vez parte de otro comentario en respuesta al anterior y así consecutivamente.

La teoría de grafos, como hemos explicado, nos sirve para esta representación. Para ello, hemos introducido en la herramienta Gephi los datos de nuestros usuarios y dado un número a cada uno para establecer una lista de nodos, por lo que el nombre de cada usuario representará un nodo. A la vez, anotamos el usuario al que respondía y la frecuencia de la interacción para obtener las aristas, es decir, las líneas de unión entre ellos. El grosor de las líneas representa el peso de cada interacción (Amat,-2014). El número máximo de interacciones entre dos usuarios ha sido de 4. Por esta razón, las aristas se muestren con grosores a cuatro escalas diferentes, en función de la frecuencia de interacción. 
Figura 1. Grafo sobre la red de usuarios y comentarios de la primera noticia analizada. El logaritmo de distribución es Force Atlas y las aristas elegidas son curvas

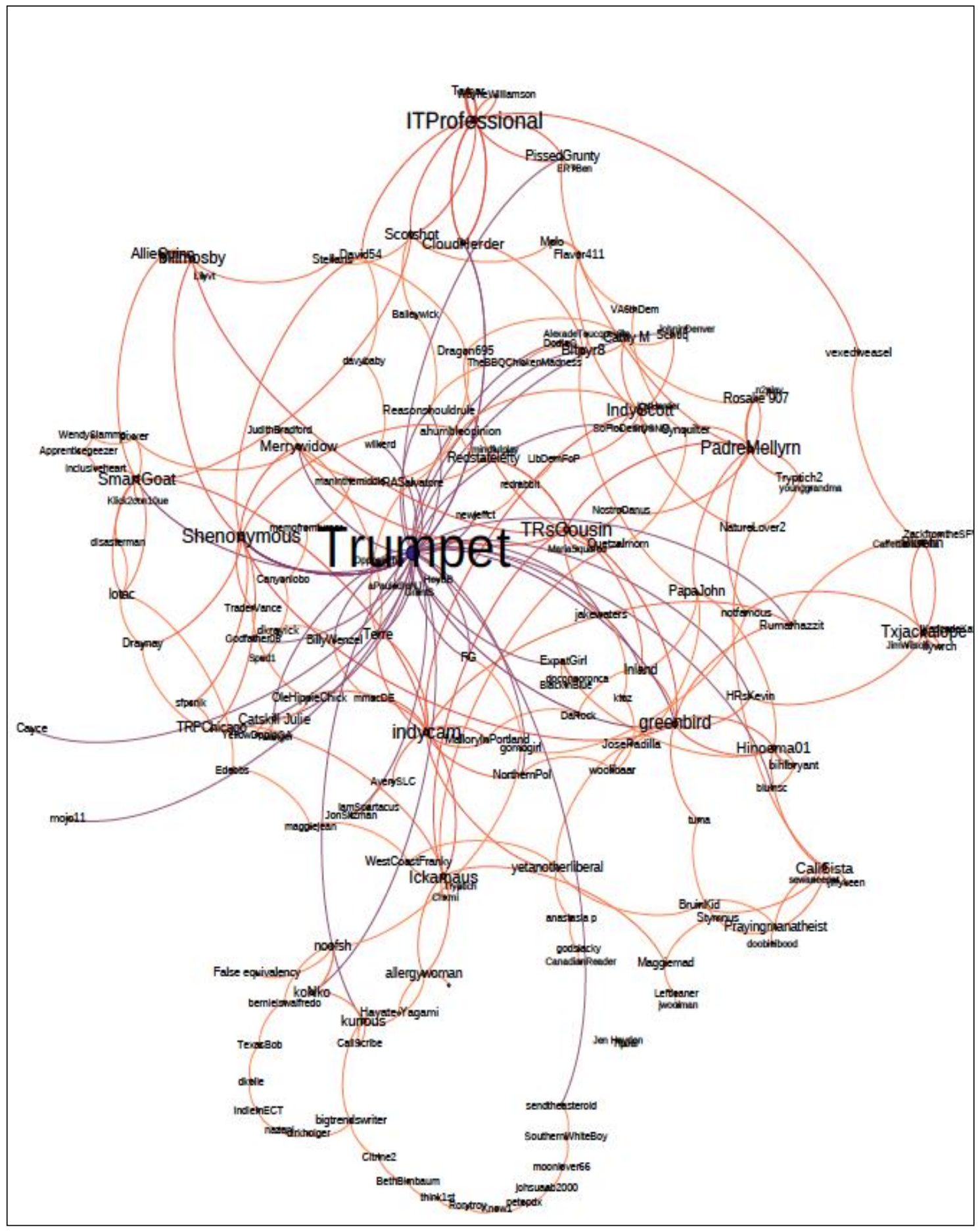

Fuente: elaboración propia

En el siguiente grafo (figura1) podemos ver la distribución de la red. En él aplicamos el algoritmo Force Atlas que da mayor protagonismo a los usuarios más influyentes o authorities, respecto a los hubs o agrupaciones de usuarios que comentan poco y, cuando lo hacen, no logran crear la polémica suficiente como para que el resto les respondan o añadan información. En total, esta gráfica representa las 303 interacciones de 165 usuarios. 
Como se puede observar, el usuario "Trumpet" aparece en el centro y un tipo de letra proporcional al peso que tiene en la conversación ya que es el que mayor número de comentarios e interacciones logra (en total: 89). Formando otros grandes centros de debate podemos ver a "IT Professional" (18 interacciones), "TRSCousin" (15), Indycam (15), "Shenonymus" (13), "SmartGoat" (13), "TRSCousin (13), "PadreMellyrn" (13), "Ickamaus" (12) y, ya con menos interacciones, "Greenbird" (11).

La mayor parte (288) de los encuestados para este trabajo (374) se consideran expresamente usuarios independientes y 210 (56\%) declararon tener 200 seguidores o más. Las excepciones más señaladas son las de algún usuario que ha contabilizado 23.000 visitas en dos años, otro que supera los 6.500 seguidores, sumando varias redes, y quienes se reconocen "activista con 4.600 seguidores en Twitter", "defensor institucional, con 400 seguidores", "profesional, con 500 seguidores" ...

La mayor parte de ellos están de acuerdo $(42,1 \%)$ o muy de acuerdo $(16,1 \%)$ con la idea de que "las redes sociales crean opinión y generan mentalidades dominantes y principios compartidos que contribuyen a determinadas percepciones sobre los grandes temas". Un $38,3 \%$ dice que no siempre sucede así ("unas veces, sí, y otras veces, no") y solo el 3,4\% restante se muestra en desacuerdo o muy en desacuerdo con la hipótesis expuesta.

\subsubsection{Facebook, la red más influyente, y Whatsapp, la más utilizada}

En esta construcción de opinión a través de redes sociales, se confirma la hipótesis segunda en el sentido de que Facebook es la red más influyente, sumando 1.211.800 engagements del total de 1.267.403 shares contabilizados en las diez noticias más compartidas durante los meses de septiembre a noviembre de 2016, en datos disponibles en Buzzsumo, que no incluye Whatsapp. Le sigue a mucha distancia Twitter, con 45.296 shares y Linkedin, con 3.569. Google+ alcanza un total de 564 y Pinterest 109. "El dominio es absoluto de Facebook en todas las noticias, superando siempre los 76.800 engagements, mientras que las demás redes sólo sobrespasan una vez los 750 shares; en concreto, Twitter, en la tercera noticia, la relativa al daño de Trump a la industria propia en beneficio de la china, en la que acumula 43.100, lejos todavía de los 126.000 de Facebook.

En nuestra encuesta, en la que cada respondente podía citar las tres más usuales, la red que aparece como más utilizada es Whatsapp (92,5\%), por lo que se acerca bastante al total. A continuación, se sitúa Facebook $(35,0 \%)$ y, a bastante más distancia, aunque muy frecuentadas también, Youtube (46\% de los encuestados) y Twitter $(36,6 \%)$. Hasta un $36,5 \%$ de las personas que respondieron a nuestra encuesta se declaran medianamente influidos y un $37,6 \%$ reconocen sentirse poco influidos. Sólo un $2,1 \%$ se considera muy influido y un 12,1\% bastante influidos. El 11,5\% asegura no sentirse nada influidos. La exposición a la influencia de las redes queda constatada por el hecho de que el $92 \%$ declara utilizarlas todos los días y el 6,4\% algunos días, quedando reducido al 1,6\% el número de quienes no las utiliza casi nunca o nunca.

\subsubsection{Veracidad e interés en las redes sociales}

El análisis de los contenidos correspondiente a las diez noticias más seguidas en redes durante el periodo estudiado confirma las cualidades de veracidad, interés y debate de ideas, que postulamos en la hipótesis tercera, al menos cuando se trata de noticias de interés internacional, como la mayoría de las diez seleccionadas. Aunque en otros casos 
los contenidos pueden llegar a ser más frívolos, se confirma que la mayor parte de los 401 comentarios seleccionados plantean debates de ideas sobre China, Trump, India, Filipinas...

Los resultados de la encuesta fijan en el $66,1 \%$ el porcentaje de quienes consideran que lo que publican las redes sociales occidentales es unas veces interesante y verdadero y otras no, a los que podrían sumarse el 5,6\% de quienes se muestran de acuerdo con la visión positiva de las redes, mientras que el 28,2 lo consideran poco interesante. El porcentaje de los que están en desacuerdo y muy en desacuerdo con la veracidad e interés de lo publicado en las redes chinas sube hasta el 32,4, abarcando el 65,1\% la ratio de quienes unas veces están de acuerdo y otras, no y quedando en el 2,5 el porcentaje de los que están de acuerdo o muy de acuerdo con su interés y veracidad.

Abundando en ello, un 33,3\% se declara en desacuerdo, más un 9,1\% muy en desacuerdo con que las redes sociales occidentales son independientes de poderes políticos y económicos, frente al $18 \%$ que cree en su independencia, permaneciendo el $38,2 \%$ restante unas veces de acuerdo y otras en desacuerdo. La desconfianza crece en el caso de las redes sociales chinas, que algunos de los encuestados afirman no conocer, elevándose al 34,8 + 21,5 el porcentaje de los que no creen que sean independientes de los poderes políticos y económicos, frente al 6,9+0,5 que sí que lo creen, quedando el $36,2 \%$ restante en posiciones intermedias.

\subsubsection{Influencias integradoras y desintegradoras}

Un buen número de investigaciones sobre los usos y abusos de los medios han demostrado la incidencia de los encuadres mediáticos y del empleo de estereotipos en los comportamientos de los ciudadanos (M. Fernández, 2013).

El estudio realizado permite validar la cuarta hipótesis en el sentido de que "los medios y agentes que operan en Internet ejercen influencias ambivalentes, contribuyendo tanto al mejor conocimiento y entendimiento entre países (influencias integradoras) como a generar desinformación, desconfianzas y recelos mediante comentarios alarmistas, uso de estereotipos, etc. (influencias desintegradoras)"; aunque habría que matizarla en el sentido de que abundan más las influencias negativas.

En las noticias y comentarios analizados destacan el gran número de textos contrarios a Donald Trump (contenidos 1 y 3 ) desde posicionamientos desintegradores: el entonces candidato es descalificado por tener productos chinos en sus hoteles y por comprar su acero, lo que podría haberse interpretado también en términos de libre mercado, de beneficio económico, etc. Los internautas aprovechan para criticar lo que consideran mala calidad de los productos chinos, salarios bajos o perjuicio para la economía. Incluso el candidato es calificado de "charlatán", "hipócrita" e "hypocritical rat-bastard". Las noticias y comentarios desde la India (contenido 2) apoyando la decisión de no comprar productos chinos, descalificándolos por el hecho de apoyar a Pakistán en sus reivindicaciones en Cachemira son asimismo posicionamientos desintegradores por la radicalidad de los mismos ("China enemy"). También se crea desconfianza, recelo e influencias negativas en la noticia y apreciaciones $\left(n^{\circ} 6\right)$ sobre la acusación de traición al senador filipino Antonio Trillanes por contactar con China y darle poder sobre los bancos de arena de los mares del sur.

Algo más integradores resultan los comentarios sobre la mujer que dijo a una familia que volviera a China (contenido 5), porque se rechaza y condena esa actitud racista y discriminatoria, y la relativa a la amistad chino pakistaní (noticia 7) en el conflicto con la 
India, aunque en el primer caso hay un trasfondo de rechazo homófobo y en el segundo una alianza contra un tercero (India). La misma intencionalidad integradora tiene la noticia o contenidos número 8 que pretende convencer a la población del interés de conocer el mandarín, aunque los comentarios de los internautas lo cuestionan y rechazan, hablando de "resultados devastadores", "mala calidad", etc. Más integrador resulta el contenido 9, en el que se justifican ayudas e inversiones chinas en Filipinas. Y en la línea ambivalente, la noticia 10 habla de colaboración entre China y Filipinas, para la compra de armas, aunque sean "para luchar contra el terrorismo".

Paradójicamente, los respondentes a la encuesta, realizada para contrastar el análisis de contenidos, suscriben, en un $84,3 \%$, que su propia influencia en redes "contribuye a un mejor conocimiento y entendimiento entre países y resulta integradora", frente al 15,6\% restante que la consideran como "fundamentalmente crítica con el statu quo y puede resultar desintegradora".

\subsubsection{Percepciones positivas y negativas sobre China}

El análisis de contenido cuantitativo y cualitativo sobre las palabras y expresiones más usadas pone de manifiesto la alta incidencia de los contextos y confirma la hipótesis en torno a que la percepción sobre China varía mucho en función de los temas tratados (muy negativa cuando se habla de negocios que dan al traste con la industria occidental y más positiva cuando se habla de colaboración con países vecinos como Pakistán y Filipinas). Es más, la palabra business tiene connotaciones negativas aplicada a China cuando se emplea en Occidente y positivas cuando se utiliza en países asiáticos. Esta variación semántica corrobora nuestro análisis en torno a los informes del Pew Research Center de Washington apuntando a que la valoración sobre China es más positiva a medida que nos acercamos geográficamente al país (con algunas excepciones como India) y más negativa cuanto más nos alejamos (algunos países europeos y americanos, aunque con bastantes excepciones).

El análisis cuantitativo de contenidos mediante la herramienta de nube de palabras Wordclouds.com (http://www.nubedepalabras.es/), aplicado a la unidad de análisis de las diez noticias sobre China más seguidas en Internet, muestra las empleadas más veces y las que pueden tener mayor interés desde el punto de vista semántico. Exceptuando, mediante el correspondiente filtrado, los términos septiembre (327), octubre (42) y noviembre (16), como meses en los que están fechadas las noticias y comentarios; los correspondientes a los protocolos de redes, como recommended (311), times (288), like (72), replay (69), partículas y nexos como just (33), can (31), will, back (29), even (29), etc., aparece como palabra más repetida el nombre de Trump $(142+22$ de Trump's), sin contar Donald (19), por la gran polémica generada en torno al uso de productos chinos en sus hoteles y por la compra de acero del mismo origen; seguida de China $(79+27$ de Chinese). El siguiente nombre propio, en plena campaña electoral a las presidenciales de Estados Unidos, fue el de Hillary (24 con 13 de Clinton) y Dana (12). Aparecen los nombres American (32+22 América), U.S. (16); people (31), York (12), States (11), United (11) ...por el americanismo denotado principalmente en la noticia sobre los hoteles de Trump, en la que se reivindica comprar productos propios. Se repiten Pakistán (21) e India (16) por el conflicto fronterizo en Cachemira.

Aparte de los nombres protagonistas, los vocablos más repetidos en estos foros y por tanto las que más interés despiertan son relativos a cuestiones económicas y empresariales; además de hotel $(47+13)$, producción, productos, trabajo y negocios: made (48), make (22), workers (24), business (22), jobs (21), companies (20+13 company), professional 
(19), works (18 +16 work), money (18), goods (16). Aparecen también new (20), way (19), media (16), room (15), steel (15), thing (14), smart (13), aluminum (12), item (12), first (12), sure (12), something (11), campaign (11), years (11). Tienen igualmente significación semántica y en este caso una connotación de oposición los pares foreign (20)/country (19) y good (27)/ bad (11). Los verbos más relevantes son los que expresan pensamiento y acción, como said (35), can (31), back (29), get (26), know (23), make (22+13 made), want (17), going (16), think (16), take (16), look (13), used (13), done (12), pay (12), bring (11), see (11). Aparecen asimismo adjetivos o adverbios de gradación como many, much...

Figura 2. Nube de palabras obtenida con la herramienta Wordclouds.com (http://www.nubedepalabras.es/)

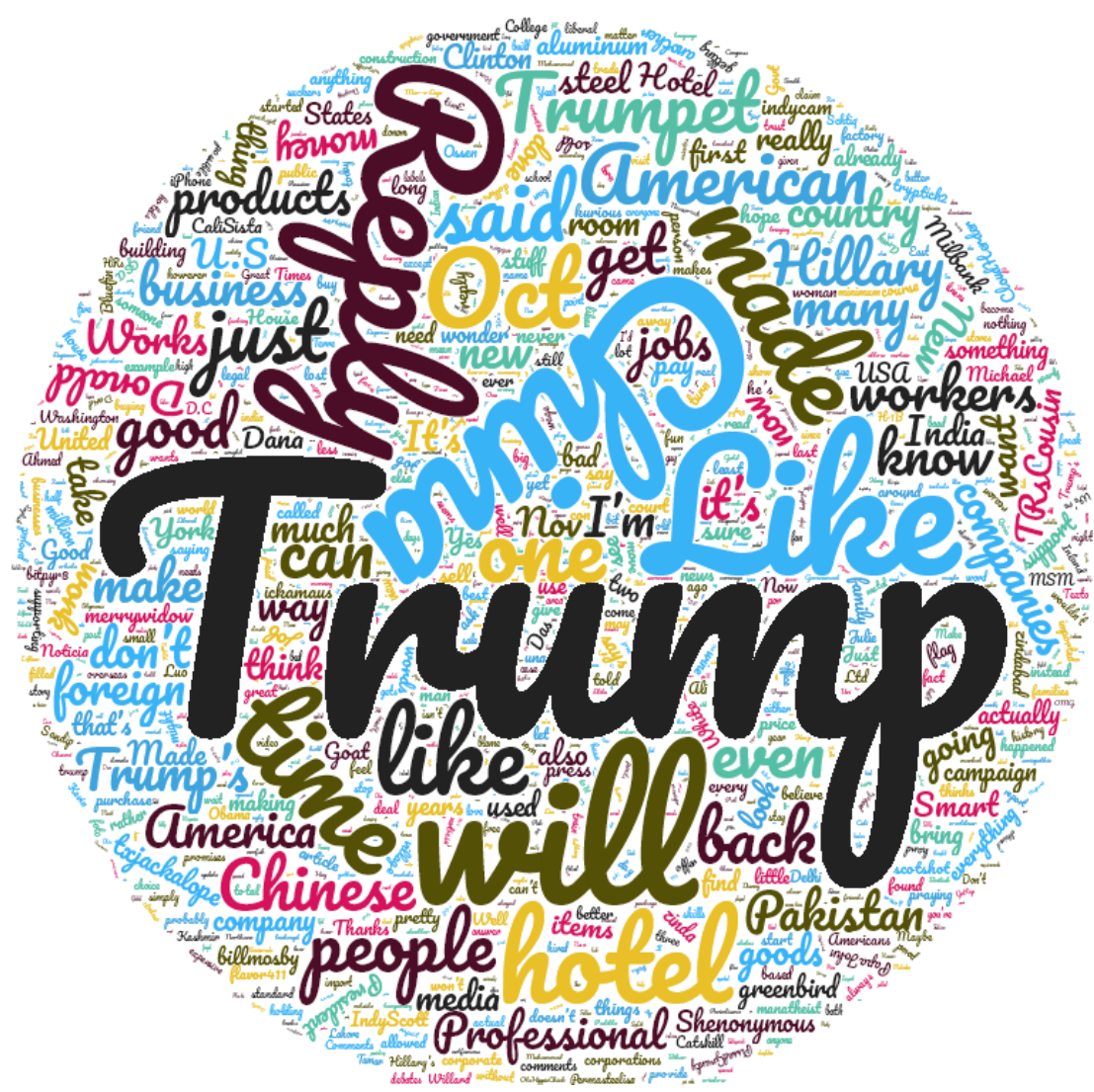

Fuente: elaboración propia

Más allá del cómputo de palabras, el análisis comprensivo e interpretativo realizado pormenorizadamente en cada una de las noticias, utilizando la Escala de Likert aplicada a las percepciones positivas o negativas sobre China, que se desprenden de cada uno de los contenidos, da como resultado, en el conjunto de la unidad de análisis, una valoración de -6 , bastante negativa, ya que predominan las valoraciones peyorativas y los estereotipos: "made in China", "goods from China", "wages as China", "oligarchs China", "China enemy", "China flooding", "Chinatown", "back to China", "fucking country", "karate and nunchucks", "China supprisses the rights", "fracking chinese colony", "difficult language", "worse quantity", "the second Persian Gulf", "history of conflict and suspicion", "aggressively moving"... En menor medida aparecen expresiones favorables: "China dosti" (amistad con China), "love China", "friendship of China", "Partnership with China", "help of China", "Chinese History", "billions from China", "relations with China"... 


\subsubsection{Análisis y contraste a partir de la encuesta}

Nuestra encuesta confirma, además de lo señalado anteriormente, que las opiniones sobre el país son favorables cuando se pregunta sobre cualidades de la población y contrarias cuando se apunta a su poderío económico y militar. El 58,3\% de los encuestados está de acuerdo en que "China es un país y una población de emprendedores y trabajadores", frente al 8,0 que se manifiesta en desacuerdo y un 38,4 que considera que unas veces es verdad y otras no. Un 30,9\% está de acuerdo (más un 2,9\% muy de acuerdo) en que "China es un país y una población pacífica, afable y colaboradora", frente al 15,6 que está en desacuerdo (más un 1,0\% muy en desacuerdo) y un 48,6\% que oscila entre ambas posturas. Pero el 35,6\% de los encuestados se preocupa porque China sea una de las mayores potencias en lo económico y militar, más un 7,5\% que está muy de acuerdo con esta preocupación, frente al $26,1 \%$ que está en desacuerdo con que el hecho sea preocupante, más un 1,9 que está muy en desacuerdo. El 28,8\% restante queda en posiciones intermedias. De forma similar los encuestados son críticos con los productos chinos. Ante la afirmación de que esos productos son de tanta calidad y fiables como los europeos, el 31,5\% se manifestó en desacuerdo, más un 9,9\% muy en desacuerdo; frente al 9,1 por ciento y 1,3 que se declararon de acuerdo o muy de acuerdo. El 48,1\% consideró que unas veces sí que son fiables y otras no.

\subsubsection{Comparación con otros estudios en torno a la percepción sobre China}

Existen paralelismos en nuestro trabajo sobre la percepción acerca de China en redes sociales con otros estudios como el del Pew Research Center de Washington que muestra que la valoración sobre China es más positiva a medida que nos acercamos geográficamente al país, aunque con excepciones en nuestra investigación, como el caso de la India, y resulta más negativa cuanto más nos alejamos (algunos países europeos y americanos, aunque con bastantes excepciones).

La valoración mayoritariamente positiva coincide asimismo con "Ratings for China Generally Favorable. Views of China", Spring 2014 Global Attitudes survey. Q15b) ${ }^{7}$, que muestra una opinión predominantemente favorable a China en América Latina. Y con el informe Transatlantic Trends, que confirma esta tendencia. Por el contrario, Ariel C. Armony y Nicolás Velásquez constatan la existencia de un discurso antichino en América Latina vinculado a su impacto sobre el medio ambiente, la migración y la demanda de recursos naturales y a su ascenso en la región.

Nuestra aportación se alinea asimismo con los trabajos de Rodríguez-Wangüemert, Rodríguez-Breijo y Pestano Rodríguez (2017), quienes observan la coexistencia de diferentes tratamientos televisivos en España sobre China como país en transición hacia el centro del contexto internacional. Los citados autores encuentran sin embargo valoraciones negativas en los canales televisivos estudiados, que promueven y mantienen recelos y prejuicios sobre su relevancia mundial (2019). De la misma forma nuestra investigación corrobora lo publicado por Arroyo-Almaraz y Van-Wyck respecto a la percepción española sobre China en el sentido de que "la población asiática, mayoritariamente procedente de China, es la más dinámica y emprendedora de todas las poblaciones inmigrantes" en el país.

\footnotetext{
${ }^{7}$ http://www.pewglobal.org/2014/07/14/.
} 


\section{Conclusiones, limitaciones y discusión}

El estudio realizado sobre la creación de opinión y percepción sobre China en redes sociales, sobre la base de las diez noticias más seguidas en los meses de septiembre a noviembre de 2016, permite apuntar, como principales resultados y con las limitaciones propias de un estudio sobre una unidad de análisis reducida, a un modelo de comunicación y de creación de opinión en redes sociales occidentales, con las siguientes características: 1. Los medios de comunicación siguen siendo los principales creadores de opinión y los que más shares y commented reciben a sus contenidos. 2. Junto a los medios aparecen influenciadores muy activos en redes (“authorities"), que contribuyen a la formación de opinión y a la generación de un debate al que se van uniendo más o menos participantes (hubs o agrupaciones de usuarios que comentan poco), según el interés despertado. Creemos que en este punto la aplicación de la teoría de grafos, rama de las matemáticas discretas, que importamos del campo de las Humanidades Digitales, ha dado muy buen resultado y puede utilizarse para seguir estudiando las conexiones que se producen en Internet en la creación de opinión. Es un modelo en el que ( $3^{\text {a }}$ característica o resultado) se duda del interés y veracidad de lo publicado en las redes, pero a la vez se reconoce el uso cotidiano mayoritario de las mismas y la existencia de significativas influencias.

La encuesta realizada a través de Google forms se ha revelado asimismo como una herramienta interesante para este trabajo, ya que ha permitido tanto corroborar muchos de los resultados del análisis como matizar otros. Y deja entrever contradicciones $\left(4^{\mathrm{a}}\right.$ característica o resultado), haciendo la comparación entre lo que los usuarios afirman de sí mismos y lo que resulta en las redes; por ejemplo, consideran que su influencia y participación son mayoritariamente integradoras, aunque resultan críticas...

Otra limitación temporal y espacial nos ha impedido desarrollar el análisis de palabras con el programa Atlas.ti y sus técnicas basadas en métodos de semántica cuantitativa. No obstante, hemos realizado un trabajo similar al propuesto por Duojiezhaxi (2016: 351) para el análisis de vocabulario: 1) palabras repetidas, es decir, frecuencias más repetidas de términos empleados en los textos, y 2) palabras clave o técnica KWICV (Key - Word in Context) para buscar los términos clave en su contexto y valor simbólico; renunciando al apartado 3 (palabras "indígenas" o nexos), por ser irrelevantes o carecer de significado semántico.

\section{Bibliografía}

Alexanderson, G. (2006). About the cover: Euler and Königsberg's Bridges: A historical view. Bulletin of the american mathematical society43(4), pp. 567-573. doi.org/10.1090/S0273-0979-06-01130-X.

Armony, A. C. y Velásquez, N. (2015). Percepciones antichinas en las comunidades virtuales latinoamericanas. Nueva Sociedad 259 (sept.-oct.), pp. 129-142. Recuperado de http://nuso.org/articulo/percepciones-antichinas-en-las-comunidades-virtualeslatinoamericanas/.

Arroyo Almaraz, I., y Van-Wyck, C. (2011). "Multicultural Marketing in Spain; perspectivas on communication directed at inmigrant population groups". Revista Latina de Comunicación Social, La Laguna, Tenerife, Universidad de La Laguna, 66, pp. 482-504. Recuperado de http://www.revistalatinacs.org/11/art/942_URJC/21_ArroyoEN.html. DOI: 10.4185/RLCS-66-2011-942-482-504-EN/ CrossRef link. 
Bastian, M., Heymann S. \& Jacomy M. (2009). Gephi: an open source software for exploring and manipulating networks. ICWSM 8, pp. 361-362. Recuperado de file:///C:/Users/Usuario/Downloads/154-3225-1-PB.pdf).

Bruns, A., Highfield, T. y Burgess, J. (2013). The arab spring and social media audiences english and arabic twitter users and their networks. American Behavioral Scientist 57(7), pp. 871-898. doi.org/10.1177/0002764213479374.

Cross, M. K., y Ma, X. (2015). EU crises and integrational panic: the role of the media. Journal of European Public Policy 22 (8), pp. 1053-1070 doi.org/10.1080/13501763.2014.984748.

Dawkins, R. [1976] 1994. El gen egoísta: las bases biológicas de nuestra conducta. Barcelona: Salvat. Recuperado de https://filosofosinsentido.files.wordpress.com/2013/07/3676.pdf.

DMR Business Statistics (2019). 70 Amazing Weibo statistics. Recuperado de http: //expandedramblings.com/index.php/weibo-user-statistics.

Duojiezhaxi (2016). Factores críticos en la comunicación de marketing online y su relación con la imagen corporativa y la mercadotecnia de relaciones en las empresas siderúrgicas chinas. Tesis doctoral. Universidad Rey Juan Carlos. Madrid.

Durkheim, É. (1893). De la division du travail social. París: Presses Universitaires de France. En español: 1987. De la división del trabajo social. Madrid: Akal.

Euler, L. (1741). Solutio problematis ad geometriam situs pertinentes. Commentarii academiae scientiarum Petropolitanae 8, pp. 128-140. Recuperado de http://eulerarchive.maa.org/pages/E053.html.

Fernández-Alameda, C. (2011), "La creación del deportista/mito en la prensa deportiva española". Tesis doctoral inédita, aprobada con sobresaliente cum laude en la Facultad de Ciencias de la Información de la Universidad Complutense de Madrid.

Fernández Fernández, M. (Ed.) (2008). Comunicación en la sociedad red: La construcción mediática de la realidad. Ávila: Universidad Católica de Ávila.

Fernández Fernández, M. (2013). La diversidad y la discriminación en encuadres de $E l$ País, El Mundo y Abc. Estudios sobre el Mensaje Periodístico, 19, pp. 91-106, Recuperado de http://dx.doi.org/105209/rev_ESMP.2013.v19.n1.42509.

Freud, S. [1921] (1979). Psicología de las masas y análisis del yo. Buenos Aires/Madrid: Amorrortu.

García Galera C., y Fernández Muñoz, C. (2016). Si lo vives, lo compartes. Cómo se comunican los jóvenes en un mundo digital. Barcelona: Ariel.

German Marshall Fund (2010). Transatlantic Trends Survey. Recuperado de http://www.gmfus.org/initiatives/transatlantic-trends-\%E2\%80\%93-public-opinion).

Golan, G. (2010). Determinants of International News Coverage. En G. Golan, T., Johnson y W. Wayne (eds). International Media Communiction in a Global Age. Nueva York: Routledge, pp. 125-144.

Halbwachs, M. (1939). Conscience individuelle et sprit collective. American Journal of Sociology 44, pp. 812-822. https://doi.org/10.1086/218172.

Halbwachs, M. [1939] (1967). La mémoire collective. París: Les Presses universitaires de France. 
Interactive Advertising Bureau (IAB) (2018). Estudio Anual de Redes Sociales 2018. Recuperado de https://iabspain.es/wp-content/uploads/estudio-redes-sociales2018 vreducida.pdf.

Katz, E. y Lazarsfeld, P. (1955). The Personal influence. The Part Played by People in the Flow of Mass Communictions. Illinois: Free Press.

Kerckhove, D. (2010). Inteligencia en conexión hacia una sociedad de la web. Barcelona: Gedisa.

Le Bon, G. [1895] 1905. La Psychologie des foules. París: Félix Alcan.

Martínez Lirola, M. (2016). Linguistic and visual strategies for portraying inmigrants as people deprived of human rights. Social Semiotics, (27 (1), 21-38.

https://www.doi.org/10.1080/10350330.2015.1137164.

McDougall, W. (1912). Psicology: The study of behavior. Nueva York: Henry Holt and Co. http://dx.doi.org/10.1037/10542-000.

Mills, Ch. W. [1959] (2004). La imaginación sociológica. México: Fondo de Cultura Económica.

Negroponte, N. (1995). Being digital. New York: Knopf.

Norris, P. (1999). ¿Quién navega? ¿La nueva tecnología transformará la democracia virtual? En Kamarck, E. (Ed) Democracy.com. Cambridge, Massachusetts: Hollis.

Pew Research Center (2014). Ratings for China Generally Favorable. Views of China. Recuperado de http://docplayer.net/9737179-Shaping-the-world-u-s-harm-many-inwith-202-419-4372-global-opposition-but-limited-america-s-image.html.

Pew Research Center (2014). China's Image. Recuperado de http://www.pewglobal.org/2014/07/14/chapter-2-chinas-image/.

Ramo, J. C. (2007). Brand China. London: Foreign Policy Centre.

Rodríguez García, T., Arroyo Almaraz, I., y Baños González, M. (2011). Análisis de la presencia de elementos narrativos en los mensajes audiovisuales utilizados por las ONG en las redes sociales. En M. Gértrudix Barrio (Ed.), Actas II Congreso Internacional Sociedad Digital: espacios para la interactividad y la inmersión. Vol. 2.

Rodríguez Wangüemert, C., Rodríguez Breijo, V., y Pestano Rodríguez, J. M. (2017). China, tras la mirada de la televisión española. Estudios sobre el Mensaje Periodístico, 23(2), 969-985, https://doi.org/10.5209/ESMP.58027.

Rodríguez-Wangüemert, C., Rodríguez-Breijo, V. y Pestano Rodríguez, J. M. (2019). Encuadres de China en la televisión española, Communication\&Society, 32(3), 123-138.

Rubio Moraga, Á. L. y Sapag Muñoz, P. (2013). Neuropolítica. El conocimiento del cerebro como base de las nuevas formas de hacer política. En Álvarez, J.T. (Ed.) Neurocomunicación. Gestión de la Comunicación Social basada en las Neurociencias, pp. 95-106. Madrid: Fragua.

Serrano-Puche, J. (2016). Internet y emociones: Nuevas tendencias en un campo de investigación emergente. Comunicar 46 (24), pp. 19-26, doi: http://dx.doi.org/10.3916/C46-2016-02.

Theilhard de Chardin, P. [1950] (1964). El grupo zoológico humano. Madrid: Taurus. 
Timoteo Álvarez, J. (2013). Neurocomunicación; el factor 'Olla de Inducción' en los procesos de Inteligencia Conectiva”. En Timoteo Álvarez, J. (Ed.) Neurocomunicación. Gestión de la Comunicación Social basada en las Neurociencias. Madrid: Ministerio de Economía y Competitividad y Grupo ThinkCom. Ed. Fragua.

Torres Nabel, L.C. (2015). ¿Quién programa las redes sociales en Internet? El caso de Twiter en el movimiento \#Yosoy132 México". Revista Internacional de Sociología, 73. http://dx.doi.org/10.3989/2013.05.29.

Transatlantic Trends (2014). Recuperado de http://trends.gmfus.org/transatlantic-trends/. Valkenburg, P., Semetko, H. y De Vrees, Claes (1999). The effects of news frames on reader's thoughts and recall. Commnunication Research, 26 (5), oct., pp. 550-560.

Syracuse: Syracuse University.

Vernadski, V. I. [1926] (1997). La biosfera. Madrid: Fundación Argentaria.

Watts, D. J. (2004). Six degrees: The science of a connected age. WW Norton \& Company.

We are social (2015). Digital, social \& Mobile Worldwide in 2018. Recuperado de https://wearesocial-net.s3.amazonaws.com/wp-content/uploads/2018/01/DIGITAL-IN2018-012-SOCIAL-MEDIA-PLATFORM-RANKING-V1.00.png).

Weber, M. [1905] (2003). La ética protestante y el espíritu del capitalismo. Madrid: Istmo.

Yeon Kim, S, Meunier, S. y Nyiri, Z. (2016). ¿Yin and yank? Public opinion in Europe toward the U.S. and China. Comparative European Politics. Macmillan Publishers Ltd.

Zhang Li (2010). In Search of Paradise: Middle Class Living in a Chinese Metropolis. Nueva York: Cornell University Press. 